\title{
Digital Holographic Method for Piezoelectric Transformers Vibration Analysis
}

\author{
Pavel Psota ${ }^{1,2}$, Václav Kopecký ${ }^{1}$, Vít Lédl ${ }^{1,2}$, Roman Doleček ${ }^{1,2}$ \\ ${ }^{1}$ Faculty of Mechatronics, Technical University of Liberec, Studentská 2, 46117 Liberec, Czech Republic \\ ${ }^{2}$ TOPTEC, Institute of Plasma Physics ASCR, Sobotecká 1660, 51101 Turnov, Czech Republic
}

\begin{abstract}
The paper describes a holographic method for vibration analysis in nanometer range. The method was successfully applied on different types of piezoelectric transformers and the measurement results are introduced. To proof the reliability of the method it was done a comparing simultaneous measurement of a piezoelectric component by the holographic method and the well-established single-point interferometric method. Results showing the minimal deviation of both methods are presented in the final part of the paper.
\end{abstract}

\section{Introduction}

A piezoelectric transformer (PT) is a device used for the transformation of alternating electric voltage by the means of ultrasonic vibrations. It utilizes converse piezoelectric effect in the input part and direct piezoelectric effect in the output part. The best transformation ratio is achieved at the mechanical vibration resonance. The measurement of out-of-plane displacement as well as the shapes of transformer vibration modes near resonant frequencies provides useful data, applicable in theoretical research of piezoelectric transformers behavior as well as in practical applications. A typical example is an effect of a transformer's properties as a function of its mechanical mounting.

PTs are used e.g. for Cold cathode fluorescent lamp electronics, high voltage generator for cold plasma, mobile phone battery recharging etc. High transformation ratio at no-load conditions is a specific feature of the PT's operation. PT applications are complex, including specific transformer mounting, wiring and driving circuit electronics. The out-of-plane displacement method is used for the PT vibration mode visualization. Studied PT's vibrate in planar modes. In-plane displacements must be re-calculated using finite element simulations.

On the market, there is a broad portfolio of measurement devices, which are usually based on the Doppler phenomenon, correlation analysis, ESPI (Electronic Speckle Pattern Interferometry) and others. Another physical principle suited for vibration analysis is digital holography (DH) $[1,2]$. The DH based methods are non-invasive, very precise and, in addition, DH enables displacement measurement over the whole-field.

The most common holographic method for vibration analysis is time average holographic interferometry, which was introduced by Powell and Stetson [3]. Unfortunately, for amplitudes smaller than approximately one tenth of the wavelength of the laser light used, the time-average method reaches its limit of minimum measurable amplitude. This problem was solved by frequency shifted holographic interferometry introduced by Aleksoff [4].

Typical analogue recording media, which was used in the past in holography, did not have uniform and repeatable response. Therefore it was impossible to determine quantitative values of amplitude precisely by the frequency shifted holographic interferometry method. The measurement usually served for estimation of vibration amplitudes and for mode structure determination.

In recent years, the resolution of CCD or CMOS have reached such a resolution, that it is possible to capture micro-interference pattern with high spatial resolution, which led to progress of $\mathrm{DH}$. $\mathrm{DH}$ became 
a powerful tool for vibration analysis in whole spectrum of measured amplitudes. However, the fringe procedure was still used $[5,6]$. Nevertheless, modern digital cameras provide the advantage of very uniform and repeatable response, and they can be considered as a radiometric detector. Vikram took this advantage and theoretically showed how to generally avoid fringe procedure with use of three digital holograms with different frequency modulations [7]. This fact also led to development of digital holographic method suitable for measurement of very small amplitudes which enables to determine quantitative values of amplitude precisely with use of only two digital hologram sequences [8]. The method is based on the application of frequency shifted digital holography with the employment of phase shifting principles. Phase shifting reduces the noise rapidly. In [8] authors introduced the basic principle and potentials of the method and the application, testing and analysis are presented in this paper. This method was successfully used for measurement of ring and disc piezoelectric transformers. The reliability of the method was tested by simultaneous measurement of uni-morph membrane for different excitation voltages by the holographic method and a singlepoint laser Michelson interferometer [9].

\section{The digital holographic method}

\subsection{Basics of digital holography}

Digital holography is whole-field and noninvasive technique with widespread applications in industry and research. Digital holography consists of two steps: the recording of the digital hologram and its reconstruction. A digital hologram is realized if the interference pattern of a reference wave and the wave reflected from the object are recorded by a digital recording medium (CCD or CMOS sensor). The resulting hologram is electronically recorded and stored. Let the diffusely reflecting object be located at a distance $\mathrm{d}$ far from the CCD. For digital reconstruction the recorded hologram $h(\Delta \xi, \Delta \eta)$ is multiplied with a numerical model of the reference wave $r(\Delta \xi, \Delta \eta)$. The field in the image plane $\mathrm{U}$ is calculated by the Sommerfeld formula which describes the diffraction of a light wave at the hologram in distance $d$ from the hologram. The Sommerfeld integral can be solved by the Fresnel approximation which in discrete finite form can be written as:

$$
\begin{aligned}
U(n, m)= & \exp \left(j \pi \lambda d\left(\left(\frac{n}{N \Delta \xi}\right)^{2}+\left(\frac{m}{M \Delta \eta}\right)^{2}\right)\right) \times \\
& \left.\times \sum_{k=1}^{N} \sum_{l=1}^{M} h(k \Delta \xi, l \Delta \eta) r \Delta \xi, l \Delta \eta\right) \\
& \times \exp \left(\frac{j \pi}{\lambda d}\left((k \Delta \xi)^{2}+(l \Delta \eta)^{2}\right)\right) \\
& \times \exp \left(-j 2 \pi\left(\frac{k n}{N}+\frac{l m}{M}\right)\right),
\end{aligned}
$$

where $j=\sqrt{-1}$ and $\lambda$ is the wavelength of the light. The stored hologram consists of $\mathrm{N} \times \mathrm{M}$ discrete values. The physical pixel size of CCD is $\Delta \xi \times \Delta \eta$ and the pixel spacing in the reconstructed image is $\Delta x \times \Delta y$. Equation (1) can be solved effectively by the FFT-algorithm. Intensity distribution $I$ and phase distribution $\varphi$ are then determined by

$$
\begin{gathered}
I(n, m)=|U(n, m)|^{2}, \\
\varphi(n, m)=\arctan (\operatorname{Im}(U(n, m)) / \operatorname{Re}(U(n, m))),
\end{gathered}
$$

where Re denotes their real part and Im denotes their imaginary part. Although the direct approach to phase distribution is a big benefit of digital holography, for vibration analysis, only the intensity field is considered.

\subsection{Principle of the method}

The experimentally easiest and the most often applied holographic method for vibration analysis is time average holographic interferometry. Let us consider a harmonically oscillating, diffusely reflecting object in position $p: d(p, t)=d(p) \sin \omega t$ where $\omega$ is the circular frequency and $\boldsymbol{d}(\boldsymbol{p})$ is the amplitude of vibration and such an oscillating object is recorded holographically using an exposure time much longer when compared to the period of the vibration of the object $T \square 2 \pi / \omega$, then the resulting intensity in the reconstructed image at position $\mathrm{p}$ is:

$$
I(p)=\mathrm{J}_{0}{ }^{2}(d(p) e(p)) .
$$

In (3) the sensitivity vector $\boldsymbol{e}(\boldsymbol{p})$ is given by the geometry of the holographic arrangement and $\mathrm{J}_{0}$ is the first kind zero-order Bessel function (see figure 1). Because there is a steady state at the nodes of the vibration modes, the intensity image exhibits the maximal intensity, $J_{0}(0)=1$ in these points. Furthermore there are dark fringes where $d(p) \boldsymbol{e}(\boldsymbol{p})$ equals the arguments of the zeros of the zero-order Bessel function of the first kind. The bright fringes 
between these zeros, which do not correspond to nodal lines, exhibit less intensity compared to the zero fringe. Thus, the nodal lines can usually be identified. The simplest example is an out-of-plane vibrating surface and a holographic arrangement with illumination and observation, normal to the surface. If each point can be assumed to oscillate only in the normal direction to the surface $\boldsymbol{d}(\boldsymbol{p})=\left(0,0, d_{Z}(p)\right)$, then $|\boldsymbol{e}(\boldsymbol{p})|=4 \pi / \lambda$ and the amplitudes at the centers of dark fringes are easily calculated by $d_{Z}(p)=b_{m} \lambda / 4 \pi$, where $b_{m}$ is the mth zero of $J_{0}$, and $\lambda$ is wavelength of the laser light used.

Unfortunately, for amplitudes smaller than approximately one tenth of the wavelength of the laser light used, the time-average method reaches its limit of minimum measurable amplitude. This problem was solved by frequency shifted holographic interferometry. In frequency shifted holography the frequency of the reference wave is modulated by an integer multiple $n \omega$ of the object vibration frequency $\omega: f_{r e f}(t)=e^{j n \omega t}$. If the object wave $U_{P}$ is not modulated and the reference wave $U_{R}$ is modulated by $f_{\text {ref }}$, the resulting intensity in the hologram plane at time $t$ is proportional to: $I(t)=\left|U_{P}+f_{\text {ref }}(t) U_{R}\right|^{2}$. Using the solution of interference holographic equation and properties of Bessel functions we obtain:

$$
I(p)=\left|U_{J n}\right|^{2}=\mathrm{J}_{\mathrm{n}}{ }^{2}(\boldsymbol{d}(\boldsymbol{p}) \boldsymbol{e}(\boldsymbol{p})) .
$$

Frequency shifted holography is used to increase sensitivity for vibrations with small as well as large amplitudes. For measurement of small amplitudes, the first order of Bessel function $J_{1}$ should be used. In time average holography with no modulation, $\mathrm{J}_{0}{ }^{2}$ in the neighborhood of zero corresponding to small amplitudes $d$ is unity and has slope of zero (the derivate is constant). Therefore for small amplitudes, it is difficult to determine significant intensity variation in the bright field, see figure 1. On the other hand, $\mathrm{J}_{1}^{2}$ in the neighborhood of zero has a positive and steep slope in the dark field yielding visible intensity variations even for small amplitudes. The slope of the higher orders (e.g. $\mathrm{J}_{2}{ }^{2}$ ) of the Bessel function is positive but not so steep. This is key to the method. The smallest detectable amplitude was estimated by Ueda, Miida and Sato [10] as $d(p) \approx \lambda / 3500$.

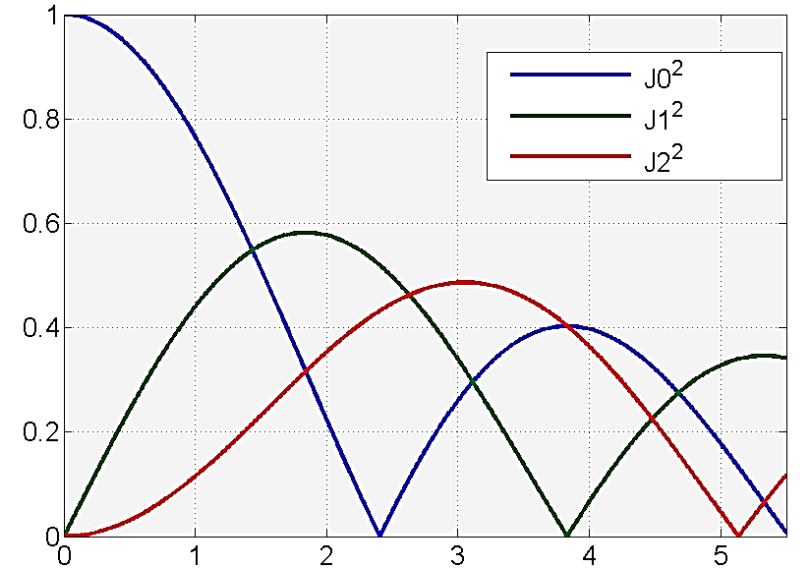

Fig. 1. $\mathrm{J} 0^{2}, \mathrm{~J} 1^{2}$ and $\mathrm{J} 2^{2}$ Bessel functions.

\subsection{Holographic setup}

Figure 2 shows the setup used, which is based on the Mach-Zehnder type of holographic interferometer. The laser beam has a wavelength of $532 \mathrm{~nm}$ and power of $100 \mathrm{~mW}$. After the mechanical shutter, the beam is split by the polarizing beam splitter, equipped with half wavelength retardation plates in two beams. Half wavelength retardation plates help set the intensities in both beams as well as the polarization of each beam. The first beam acts as a reference wave and could be further attenuated if necessary by a set of gray filters placed in filter wheels. Each beam is frequency shifted by an acousto-optic frequency modulator - Bragg cell, with a fundamental frequency of $40 \mathrm{MHz}$. The beams are spatially filtered and collimated. After two reflections on mirrors M2 and M3, the reference beam hits CCD. The object beam illuminates the sample and the light scattered from its surface impinges on the CCD sensor. The setup is designed as an off axis LeithUpatnieks scheme. The angle between the beams is set to be approximately $3^{\circ}$. The camera is an AVT Stingray - F 504 with a resolution of $2045 \times 2056$ pixels, each pixel having the size $3.45 \times 3.45 \mu \mathrm{m}$. The camera is connected to the computer via a Fire Wire B interface enabling a frame rate of 6.5 FPS. The image from camera digital hologram - is cropped to $2048 \times 2048$ pixels. A sequence of 16 frames was captured. 


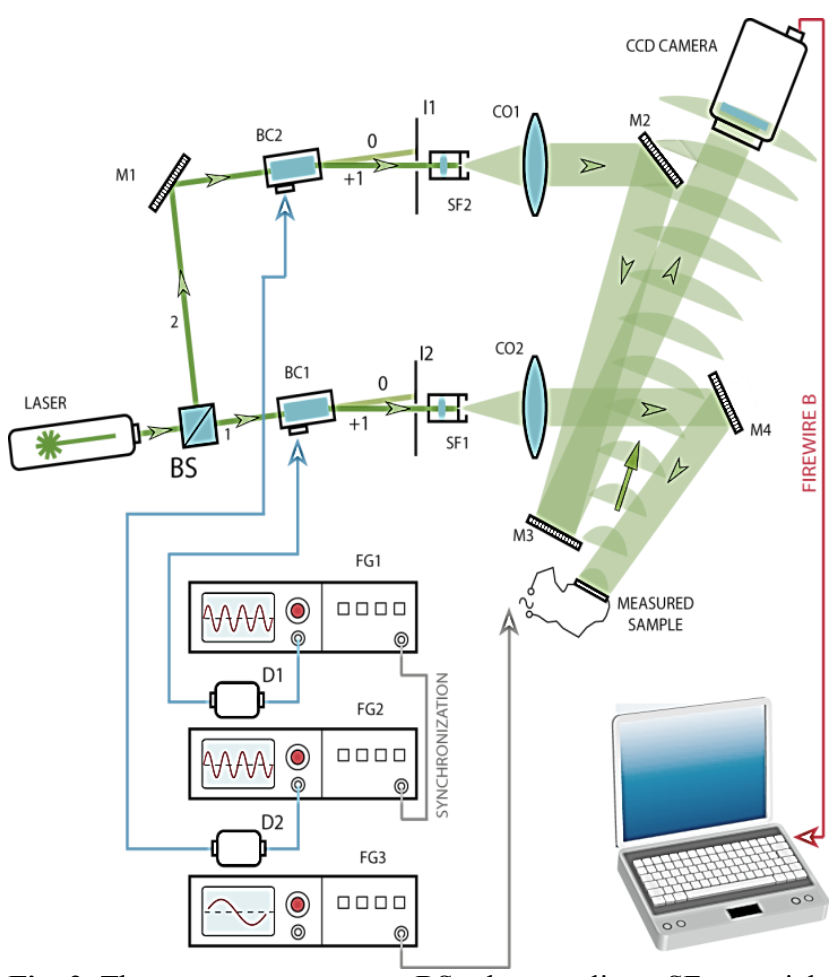

Fig. 2. The measurement setup: $\mathrm{BS}$ - beam splitter, $\mathrm{SF}$ - spatial filter, $\mathrm{BC}$ - Bragg cell, $\mathrm{M}$ - mirror, $\mathrm{CO}$ - collimating objective, $\mathrm{FG}$ - function generator, $\mathrm{D}$ - driver.

\subsection{Data evaluation}

The method for piezoelectric transformer measurement is based on the application of frequency shifted digital holography with the employment of phase shifting principles [8]. Frequencies of Bragg cells are chosen to fulfil the equation

$$
\Omega_{B 2}=\Omega_{B 1}+\omega+\omega_{C} / M,
$$

where $\omega$ is the frequency of the object vibration $\omega_{C}$ is the camera's frame frequency and $\mathrm{M}$ is number of frames used in heterodyne detection. This way, a sequence of $\mathrm{M}$ phase shifted digital holograms is recorded and processed by formula

$$
h=1 / M \sum_{m=0}^{M-1} h_{m} e^{2 \pi m / M}
$$

to get a filtered digital hologram h. Afterwards, the processed hologram is reconstructed using Fresnel transform (1) and the intensity distribution is calculated with use of formula (2). The interferogram reconstructed from the processed hologram $h$ has a much higher signal to noise ratio and strongly suppressed DC term compared to the interferogram reconstructed from a single hologram. To suppress speckle noise in interferograms generated by coherent illumination, some advanced filtering methods can be used [11]. Low overall noise is a key factor to get the very high resolution of this method. Since the reflectance of the piezoelectric transformer is not uniform along the entire surface, the reference interferogram with no frequency shift of the reference wave in a steady state must also be recorded. Such an interferogram represents the $J_{0}$ intensity distribution of the steady state. The reference interferogram $\mathrm{U}_{\mathrm{J} 0}$ is used to normalize the interferogram $\mathrm{U}_{\mathrm{J} 1}$, reconstructed from the digital hologram with a frequency shifted reference wave: $U_{J 1 \text { norm }}=U_{J 1} / U_{J 0}$. A necessary condition for normalization is uniform response of the recording medium during the entire recording. Modern digital cameras meet these requirements. On the other hand, typical analogue recording media, being used in past in holography, do not have this property and therefore are not suitable for this method. After the normalization operation, we get a normalized version of (4): $U_{J 1 \text { norm }}(p)=\mathrm{J}_{1}(\boldsymbol{d}(\boldsymbol{p}) \boldsymbol{e}(\boldsymbol{p}))$. In our experimental setup the sensitivity vector is approximately perpendicular to the CCD target and its magnitude is $|\boldsymbol{e}(\boldsymbol{p})|=4 \pi / \lambda$. Therefore the out-of-plane vibration amplitude $d_{Z}$ in every surface point $p$ can be directly calculated by the inversion of the first monotonic interval of:

$$
U_{J 1 \text { norm }}(p)=J_{1}\left(4 \pi d_{Z}(p) / \lambda\right)
$$

For the inverse transfer function, it is necessary to determine the out-of-plane vibrations amplitude corresponding to $J_{I M A X}$. The normalization of $U_{J l}$ permits us to use well defined relations between $\mathrm{J}_{0}$ and $\mathrm{J}_{1}$ functions:

$$
J_{1 M A X}=0.582 J_{0}(0) \text { and } J_{1 M A X}=J_{1}(1.84)
$$

Thus we can derive the maximum amplitude: $d_{M}=1.84 \lambda / 4 \pi$. For our experiment, the maximum amplitude is $d_{M}=77,8968 \mathrm{~nm}$ and the transfer function is displayed in figure 3 . This maximum amplitude $d_{M}$ is also the limiting value for the maximal measured amplitude by this method, without the risk of ambiguities. For greater amplitudes, standard time average digital holography, represented by the $\mathrm{J}_{0}$ function, serves perfectly.

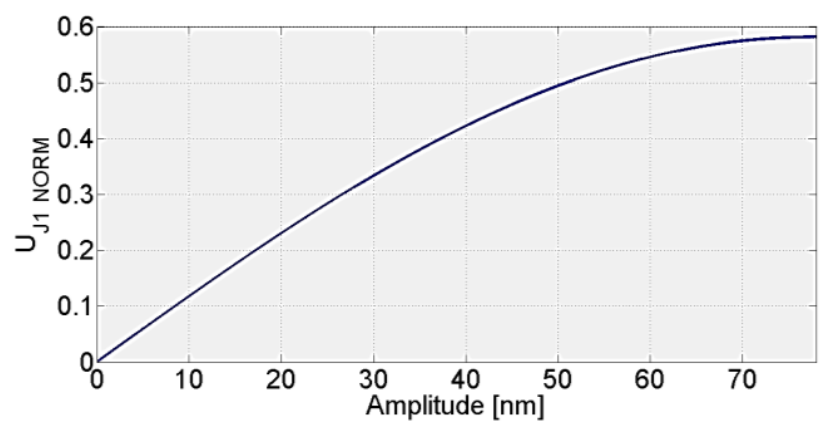

Fig. 3. The transfer function for the calculation of amplitudes.

\section{Measurement results}

The measured samples were ring PT with a diameter of $20 \mathrm{~mm}$ and a thickness of $1 \mathrm{~mm}$. Electrodes were 
designed in 4-segments shapes with different wedge angles. The PTs were placed in a manufactured holder. The disc was pushed by a spring from the back-side to ensure that the pressure would be approximately identical during all measurements. The PTs were driven by a voltage of $30 \mathrm{~V}_{\mathrm{pp}}$. Some results of disc PT measurement are shown in figure 4. The intensity of pseudo-color image corresponds to the amplitude of the vibration according to the color-bar on the right-hand side. The dark black lines can be considered as nodal lines where the vibration amplitude is zero. Since the measurement method is based on modified time average holography, displacement magnitude of an oscillation from an equilibrium position is measured and it is not possible to measure the phase of vibrations.

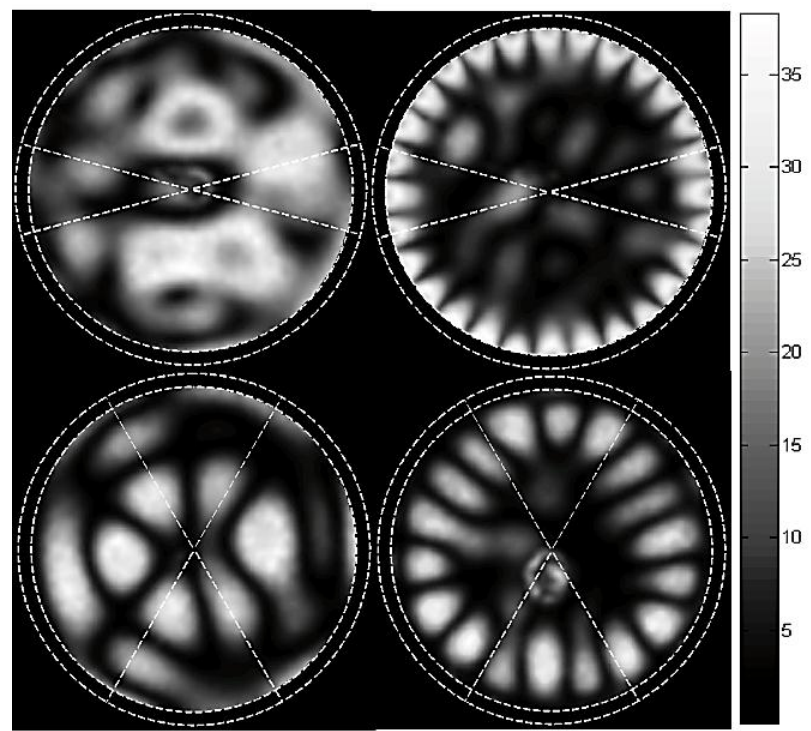

Fig. 4. The out-of-plane displacement (all units of amplitudes are in nanometers) of disc piezoelectric transformers with 4-segment electrodes shape. The $30^{\circ}$ wedge 4-segment electrodes shape PT for resonant frequencies $118.70 \mathrm{kHz}$ (left) and $251.55 \mathrm{kHz}$ (right) driven by $30 \mathrm{~V}_{\mathrm{pp}}$. The 4 -segement $120^{\circ}$ electrodes shape PT for resonant frequencies $140.65 \mathrm{kHz}$ (left) and 249.70 $\mathrm{kHz}$ (right) driven by $30 \mathrm{~V}_{\mathrm{pp}}$. Total transformer diameter is $20 \mathrm{~mm}$, dashed white lines represent position of borderlines between electrode segments.

The reliability of this method was tested by the simultaneous measurement of the uni-morph membrane for different excitation voltages by the holographic method and using a single-point laser Michelson interferometer $[9,12]$. With the use of a lock-in amplifier technique, the Michelson interferometer enables us to measure amplitudes in the order of $10^{-12} \mathrm{~m}$. In part, we wanted to compare the amplitude values of a measurement point obtained by these methods, while, on the other hand, to confirm our prediction that the method is linear. Although the holographic method is a whole-field method, whereas laser interferometry can measure amplitude in single point only, it was necessary to consider holographic data only for the area of interest. The results are plotted in figure 5 and figure 6 . It seems the measurement error increases with smaller vibration amplitude. However, it can be seen that the estimated relative difference is fewer than $10 \%$. The reliability of the holographic method for measurement of extremely small amplitudes (sub-nanometers) is under investigation now.

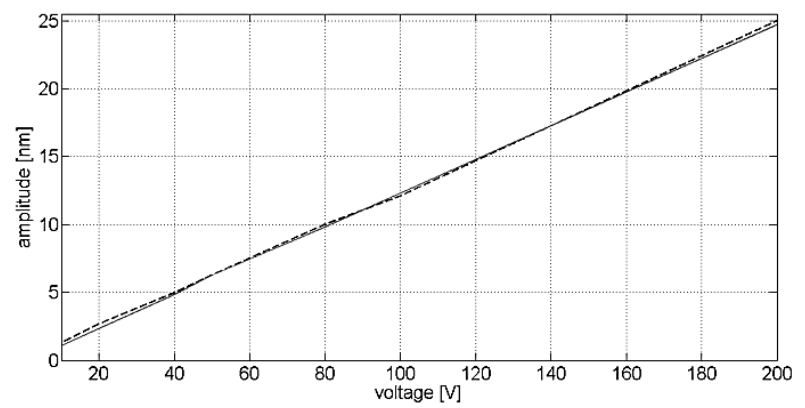

Fig. 5. Simultaneous amplitude measurement by holographic (full line) and interferometric (dashed line) method for different excitation voltages (left).

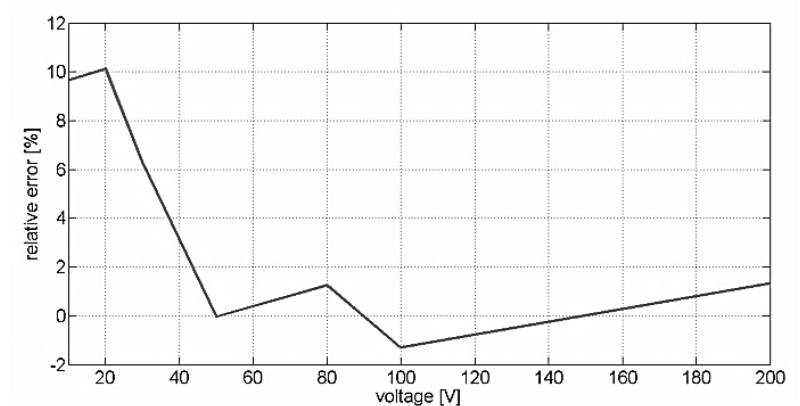

Fig. 6. The relative percentage deviation of holographic method from the interferometric method.

\section{Conclusion}

Time average holographic interferometry, together with the properties of modern electronic cameras, (especially their linearity and exposure stability) combined with computer processing, allow for vibration measurement with amplitude and resolution in a sub-nanometer scale. The method for the direct determination of absolute amplitude of vibration from intensities in reconstructed holograms modulated by $\mathrm{J}_{1}$ (first kind zero order Bessel function) was developed. At least two exposures are necessary. Reaching nanometer and sub-nanometer scale imposes high requirements on signal to noise ratio in reconstructed holograms. Phase shifting heterodyne time average holographic interferometry was used to acquire almost noise-free holograms. The combination of all those techniques 
allowed measurement of the vibration of piezoelectric transformers in nanometer and subnanometer scale in the whole area. The measurement was done for resonant frequencies of ring and disc transformers driven by various voltage amplitudes. Moreover, the results of this developed method were compared to the measurement results of a single-point Michelson interferometer. It was demonstrated that this method is linear and reliable in the whole measurement range. The estimated measurement error of the method was estimated to $0.1 \mathrm{~nm}$.

\section{Acknowledgements}

This work was supported by the Student grant scheme at the Technical University of Liberec and by the European Regional Development Fund and the Ministry of Education, Youth and Sports of the Czech Republic in the Project No. CZ.1.05/2.1.00/03.0079: Research Center for Special Optics and Optoelectronic Systems (TOPTEC).

\section{References}

1. T. Kreis, Handbook of Holographic Interferometry, (2004).

2. R.L. Powell and K.A. Stetson, J. Opt. Soc. Am. 55, 1593-1598 (1965).

3. C. C. Aleksoff, Appl. Opt. 10, 1329-1341 (1971).

4. Y. Ishii, Optics and Lasers in Engineering, 36, (2001).

5. F. Joud, F. Laloë, M. Atlan, J. Hare, and M. Gross, Optics express, 17, 2774-2779 (2009).

6. Ch. Vikram, Journal of Modern Optics, 39, (1992)

7. V. Lédl, J. Václavík, R. Doleček and V. Kopecký, AIP Conf. Proc. 1253, (2010).

8. M. Šulc, Interferometer Based Methods for Research of Piezoelectric Materials in Interferometry Principles and Applications, (2011). 